\title{
Lightweight Energy Management of Islanded Operated Microgrids for Prosumer Communities
}

\author{
Riccardo Bonetto, Tommaso Caldognetto, Simone Buso, Michele Rossi, Stefano Tomasin, Paolo Tenti \\ Dept. of Information Engineering (DEI), University of Padova, Via G. Gradenigo 6/B, 35131 Padova (PD), Italy \\ \{name.surname\}@dei.unipd.it
}

\begin{abstract}
We present and evaluate a lightweight and effective approach for the management of prosumer communities through the synergistic control of the power electronic converters acting therein. These controllable elements are the utility interface (UI), installed at the point of common coupling with the electrical utility, and the energy gateways (EGs), interfacing distributed generation units and energy storage devices with the distribution grid. The UI acts as the control master for the microgrid, collecting information on generators and power demand and dispatching a control parameter that regulates both energy storage devices and generators. An islanded operation mode is considered, and the control strategy aims at leveling peaks in the energy drained from or injected into the UI. The proposed control strategy is tested on a residential microgrid model, $100 \mathrm{kVA}$ rated, which has been developed and utilized to analyze selected performance metrics in the presence of realistic and time varying power demand and energy generation processes. This model allows a fine-grained analysis of a) the energy storage state at each residential unit, b) the amount of energy required at the UI, and c) the locally generated energy injected into the micro grid by each EG. As a further result, our framework returns the amount of distributed storage required to achieve a target peak-shaving performance level, according to the number of residential users with generation capability and their storage capacity.
\end{abstract}

\section{INTRODUCTION}

$\mathrm{L}$ OW-VOLTAGE MICROGRIDS will play a major role in future smart grids [1]. The presence of distributed microgeneration and energy storage owned by end users (referred to here as prosumers) results in a new paradigm for electrical grids and in a potentially new and vibrant market for technology manufacturers, service providers, energy traders, distributors, and regulatory boards. However, several challenges are still to be faced, in terms of technology, standards, rules, and economic models [2], [3]. According to this new paradigm, the distribution grid can be seen as a patchwork with the microgrids being its basic tiles and supporting the utility in terms of power quality, management of network dynamics, etc. Also, microgrids could be engineered so as to assure electrical continuity to the loads even in the case of grid failure. A major goal of microgrids is to integrate and effectively manage distributed energy resources (DERs), either as micro-generation (MG) or energy storage (ES). In fact, the increasing pervasiveness of renewable energy sources, mainly photovoltaic (PV), may lead to the misbehavior of the distribution grid due to over-production during daytime, while having a negative impact on the electrical market. Thus, the capability to control the energy in- and out-flow of microgrids, seen as an aggregate of entities (prosumer communities), plays a major role in ensuring stability, efficiency and cost-effectiveness of future smart grids. Toward this objective, each energy gateway (EG, i.e., the controller placed at the user' premises) must be properly driven, and the control architecture must be flexible and scalable, so as to accommodate any number of DERs and autonomously adapt to the power variations due to the loads and to the intermittent energy sources [4]-[10].

In this paper, we propose a lightweight control approach to realize this vision, extending the work of [11] through a control architecture capable of guaranteeing the correct operation of prosumer communities in an islanded operated scenario. In detail, the proposed control strategy adopts a master-slave approach, where the master role is played by the utility interface (UI), i.e., a three-phase inverter located at the point of common coupling (PCC) between the microgrid and the utility. The UI is equipped with energy storage (battery or super-cap) and, if necessary, with a backup generator (such as a microturbine, fuel cell, diesel gen-set, etc.), permanently performing as a voltage source. We assume that the microgrid operates in islanded mode, the UI acts as a grid-forming voltage source, as the mains, while the EGs act as current sources [11]. The purpose of the control strategy is to level peaks in the user demand, thus limiting the stage/production requirements to the UI. We achieve this goal by a semi-distributed approach, where the UI collects information on load and source activity and distributes a single control parameter that is then used locally to regulate the operation of ES and generators.

Note that an islanded operation mode entails an additional number or issues, including frequency and voltage control, which are not within the scope of this paper. Here, we assume that some other control strategy takes care of these issues by operating at a suitably fast rate. Instead, our control operates at a slower rate (addressing slower variations of loads and sources) with the aim of ensuring the long-term sustainability of the microgrid.

A major advantage of our approach is the additional degree of freedom gained in the internal optimization of the microgrid, which is now seen by the utility as an aggregate user, with improved efficiency and control capabilities. Note that this might create new market opportunities and monetization strategies, since prosumer communities could upgrade their role and increase their contracting clout, by taking advantage of autonomous management. As a by-product, we are also able to control the microgrid when operating in a grid-connected mode, with the UI behaving as a grid-interactive UPS, and 


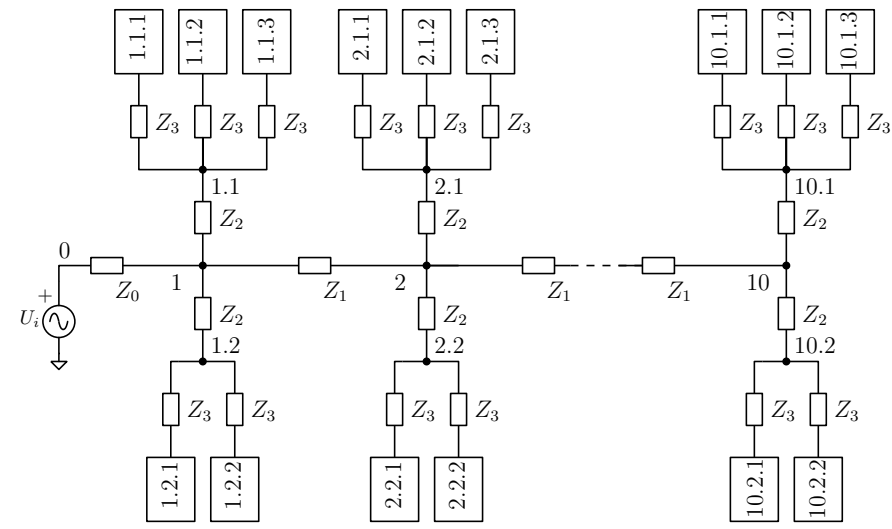

Fig. 1. Schematic representation of the considered microgrid topology.

playing the role of the central controller for the microgrid.

To validate the proposed control strategy, a residential microgrid model, $100 \mathrm{kVA}$ rated, is developed to simulate realistic power demand and energy generation processes. This model integrates real life data regarding power consumption (demand) and photovoltaic generation, featuring validated statistical models for both processes. Thanks to these tools, we evaluate the impact of the proposed control strategy (dealing with peak shaving at different time scales) on the performance of the microgrid and assess its peculiarities in terms of required energy storage and load balancing capabilities. We note that the considered statistical approach for the energy sources and the loads leads to a more realistic system design methodology than considering, e.g., worst case scenarios.

The remainder of this paper is structured as follows. In Section II we describe the system model, whereas our control algorithm is presented in Section III. Simulation results are presented in Section IV and our conclusions are drawn in Section V.

\section{SySTEM MODEL}

We consider a low voltage, single phase residential microgrid, schematically represented in Fig. 1, with $K=50$ end user nodes. At the PCC (node 0), the microgrid is equipped with a distinct unit, denoted three-phase utility interface (UI) with energy storage (UI-ES) capabilities and power capability of $100 \mathrm{kVA}$. Downstream from the PCC, the grid is composed of 10 topologically identical sections, each comprising 5 end users. The electrical network is represented by a tree with three levels of depth, with the end-users being its leaves (in Fig. 1, end-users are univocally identified by a triplet "i.j.k"). Each tree level is characterized by a characteristic interconnection impedance. As a result, besides the UI output impedance $\left(Z_{0}\right)$, three additional interconnection impedances are considered, as reported in Tab. I.

The UI is controlled as a voltage source and is capable of bidirectional communication (via power line or wireless) with any other node of the microgrid. $N=15$ grid nodes, randomly distributed within the microgrid, are active nodes with micro-generation and energy storage capabilities. These nodes are interfaced to the grid through an energy gateway
TABLE I

MICROGRID INTERCONNECTION IMPEDANCES

\begin{tabular}{llrl}
\hline Tree level & Value & Unit & \\
\hline Zero level (PCC) & $Z_{0}$ & $+j 26$ & $\mathrm{~m} \Omega$ \\
Level 1 & $Z_{1}$ & $173+j 44$ & $\mathrm{~m} \Omega$ \\
Level 2 & $Z_{2}$ & $267+j 75$ & $\mathrm{~m} \Omega$ \\
Level 3 & $Z_{3}$ & $705+j 157$ & $\mathrm{~m} \Omega$ \\
\hline
\end{tabular}

(EG), which operates as a current source and is capable of bidirectional communication with the UI. The remaining $M=K-N=35$ nodes are passive nodes. Passive nodes, although possibly equipped with smart meters (SM), are not necessarily endowed with intelligent measurement or control devices. In grid-connected mode, the UI voltage reference is set by suitable active and reactive power control loops, while in islanded mode, the UI becomes the grid-forming voltage source for the entire microgrid. The EGs make their energy resources available, including their local energy storage. The active nodes perform as slaves and their EGs communicate with the UI, implementing distributed control actions. Although different power definitions can be used within the proposed approach, we consider conservative quantities for the active and reactive power, see [12]. Also, without loss of generality, we refer to single-phase variables, being aware that single-phase and three-phase loads may coexist in the same microgrid.

In grid-connected operation, the UI only supplies reactive power to perform ancillary functions, like power factor control at the PCC and load imbalance compensation. Moreover, the UI dispatches active and reactive power commands to the EGs. The EGs, in turn, make their residual power capability available to the UI, and possibly perform as active filters to mitigate current distortion. In grid-connected mode, any error or delay in the power commands dispatched to EGs is non time-critical, since the power balance is ensured by the mains, at any time and in any condition. Instead, in islanded mode the UI becomes the voltage source for the entire microgrid, and makes use of its ES and/or backup unit to maintain the PCC voltage at the desired level. The power references dispatched to current-controlled EGs become then time-critical, since the power balance within the microgrid must now be autonomously provided by the EGs and the UI. Therefore, suitably fast-rate control strategies must be deployed to ensure stability. Here we do not address this fast control. Rather, we focus on a longer-term control that avoids long lasting overconsumption. A degree of freedom is offered by load control, if any. In fact, if the power balance cannot be ensured by the available energy sources, the UI can ask the EGs to disconnect some low-priority loads to reduce the power demand.

\section{MICROGRID CONTROL STRATEGY}

Next, we describe how the microgrid can be controlled in grid-connected and islanded operation modes. At the beginning of every control period $T_{S}$ (whose duration is a few line cycles) the UI, as the control master, polls all the nodes of the 
microgrid. The active nodes return the values of active and reactive power available for microgrid control. Note that the number of active and passive nodes can dynamically change, depending on how many end-users are actually connected to the microgrid. Moreover, active nodes perform as passive ones when their generated power is fully used to feed their local loads. Therefore, the control algorithm must be devised to allow dynamic adjustment of microgrid parameters. In detail, the data packet sent by the $n$-th EG (slave unit) to the UI (master controller) at the end of the $\ell$-th control cycle includes:

- $P_{G n}(\ell), Q_{G n}(\ell)$, the active and reactive power generated by the local power source during the $\ell$-th cycle,

- $P_{G n}^{\max }(\ell+1)$ and $P_{G n}^{\min }(\ell+1)$, the estimate of the upper $(\max )$ and lower $(\min )$ limits of the active power from the local energy source in cycle $\ell+1$, also taking into account the power that can be fed into $\left(P_{S n}^{\mathrm{in}}\right)$ or drained from $\left(P_{S n}^{\text {out }}\right)$ the local ES. Since $P_{S n}^{\text {out }}>0$ and $P_{S n}^{\text {in }}<0$, it holds:

$$
\begin{aligned}
& P_{G n}^{\min }(\ell+1)=P_{G n}(\ell)-P_{S n}^{\mathrm{in}}(\ell+1), \\
& P_{G n}^{\max }(\ell+1)=P_{G n}(\ell)+P_{S n}^{\text {out }}(\ell+1) ;
\end{aligned}
$$

- $P_{G n}(\ell+1)$, the estimate of the active power that will be generated in the $\ell+1$-th cycle,

- $A_{G n}(\ell+1)$, the estimate of the nominal power flow capability of the EG, and

- $A_{G n}^{\text {over }}(\ell+1)$, the estimate of the overload power flow that can be temporarily sustained by the EG (e.g., for $10-100$ grid cycles). The overload power rating is related to the instantaneous physical capability of the energy gateway. This parameter can vary in time, for example, as a consequence of thermal stresses.

Finally, the UI determines the active and reactive power, $P_{t o t}^{P C C}(\ell)$ and $Q_{t o t}^{P C C}(\ell)$, respectively, absorbed by the microgrid from the PCC and measured during the $\ell$-th cycle.

To derive the subsequent control actions, the UI estimates the energy state of the microgrid by computing, on the basis of the collected data, the following quantities:

- the total power generated by the EGs in the $\ell$-th cycle:

$$
P_{G t o t}(\ell)=\sum_{n=1}^{N} P_{G n}(\ell), \quad Q_{G t o t}(\ell)=\sum_{n=1}^{N} Q_{G n}(\ell),
$$

- the total power absorbed by the loads in the $\ell$-th cycle:

$$
\begin{aligned}
P_{\text {Ltot }}(\ell) & =P_{\text {tot }}^{P C C}(\ell)+P_{\text {Gtot }}(\ell), \\
Q_{\text {Ltot }}(\ell) & =Q_{\text {Ltot }}^{P C C}(\ell)+Q_{\text {Gtot }}(\ell),
\end{aligned}
$$

- the estimated power absorbed by the loads in the next cycle $\ell+1$ :

$$
\begin{aligned}
& P_{L t o t}(\ell+1)=P_{L t o t}(\ell)-P_{G 0}(\ell+1), \\
& Q_{L t o t}(\ell+1)=Q_{L t o t}(\ell)-Q_{G 0}(\ell+1),
\end{aligned}
$$

where $P_{G 0}(\ell+1)$ and $Q_{G 0}(\ell+1)$ are the estimates of the active and reactive power that the UI expects to generate in the next control cycle $\ell+1$.
- the expected available active and reactive power, in normal or overload conditions, from the distributed EGs in the next control cycle $\ell+1$ :

$$
\begin{gathered}
P_{G t o t}(\ell+1)=\sum_{n=1}^{N} P_{G n}(\ell), \\
P_{G t o t}^{\min }(\ell+1)=\sum_{n=1}^{N} P_{G n}^{\min }(\ell+1), \\
P_{G t o t}^{\max }(\ell+1)=\sum_{n=1}^{N} P_{G n}^{\max }(\ell+1), \\
Q_{G n}^{\max }(\ell+1)=\sqrt{A_{G n}(\ell+1)^{2}-P_{G n}(\ell+1)^{2}}, \\
Q_{G t o t}^{\max }(\ell+1)=\sum_{n=1}^{N} Q_{G n}^{\max }(\ell+1), \\
Q_{G n}^{\text {over }}(\ell+1)=\sqrt{A_{G n}^{\text {over }}(\ell+1)^{2}-P_{G n}(\ell+1)^{2}}, \\
Q_{G t o t}^{\text {over }}(\ell+1)=\sum_{n=1}^{N} Q_{G n}^{\text {over }}(\ell+1) .
\end{gathered}
$$

Then, the above estimates are used by the power-based control algorithm to determine the power contributions of the distributed generators.

The amount for power injected by the active nodes is finally obtained by means of two variables, $\alpha_{P}$ and $\alpha_{Q}$, calculated by the UI and then broadcast to all EGs. In the following paragraphs, we describe how these coefficients are determined for the various operating modes.

\section{A. Islanded Operation - Active Power}

Next, we consider four cases, that are related to the amount of predicted power generation/consumption at the next cycle.

1) $P_{\text {Ltot }}(\ell+1)<P_{G t o t}^{\min }(\ell+1)$ : in this case, the aggregated power demand $\left(P_{L t o t}(\ell+1)\right)$ is smaller than the minimum power that can be generated by the active nodes, although local accumulators are fully exploited. Thus $\alpha_{P}=0$. Correspondingly, each active node generates a power reference:

$$
P_{G n}^{*}(\ell+1)=P_{G n}^{\min }(\ell+1) .
$$

In case the total injected power exceeds the power demand, the surplus is stored in the UI accumulators, so as to assure the power balance of the microgrid.

2) $P_{\text {Gtot }}^{\min }(\ell+1) \leq P_{\text {Ltot }}(\ell+1)<P_{\text {Gtot }}(\ell+1)$ : here, the power absorbed by the loads can be directly provided by the active nodes. We set:

$$
\alpha_{P}=\frac{P_{\text {Ltot }}(\ell+1)-P_{G t o t}^{\min }(\ell+1)}{P_{\text {Gtot }}(\ell+1)-P_{\text {Gtot }}^{\min }(\ell+1)}, \quad 0 \leq \alpha_{P} \leq 1 .
$$

Correspondingly, each active node generates an active power reference equal to:

$$
\begin{aligned}
P_{G n}^{*}(\ell+1) & =P_{G n}^{\min }(\ell+1)+ \\
& +\alpha_{P}\left(P_{G n}(\ell+1)-P_{G n}^{\min }(\ell+1)\right) .
\end{aligned}
$$


3) $P_{\text {Gtot }}(\ell+1) \leq P_{\text {Ltot }}(\ell+1) \leq P_{\text {Gtot }}^{\max }(\ell+1)$ : the power absorbed by the loads can be delivered by the active nodes with the support of the distributed ES. Also in this case, the UI does not necessarily exchange active power with the grid, although it can restore the state of charge of its ES by summing the additional power to the load power. We set:

$$
\alpha_{P}=1+\frac{P_{L t o t}(\ell+1)-P_{G t o t}(\ell+1)}{P_{G t o t}^{\max }(\ell+1)-P_{G t o t}(\ell+1)}, \quad 1 \leq \alpha_{P} \leq 2 .
$$

Correspondingly, each active node generates an active power reference equal to:

$$
\begin{aligned}
P_{G t o t}^{*}(\ell+1) & =P_{G n}(\ell+1)+ \\
& +\left(\alpha_{P}-1\right)\left(P_{G n}^{\max }(\ell+1)-P_{G n}(\ell+1)\right) .
\end{aligned}
$$

4) $P_{\text {Ltot }}(\ell+1)>P_{\text {Gtot }}^{\max }(\ell+1)$ : the power demand exceeds the maximum power that can be generated within the microgrid, even though distributed ES were fully exploited. In this case we set $\alpha_{P}=2$. Correspondingly, each active node generates an active power reference equal to:

$$
P_{G n}^{*}(\ell+1)=P_{G n}^{\max }(\ell+1) .
$$

In case the total power injected by the EGs is insufficient to satisfy the loads, the needed additional power is drained from the UI.

\section{B. Islanded Operation - Reactive Power}

1) $Q_{\text {Ltot }}(\ell+1) \leq Q_{\text {Gtot }}^{\max }(\ell+1)$ : here, the reactive power can be delivered by the distributed EGs without overloading their power interfaces. In this case we set:

$$
\alpha_{Q}=\frac{Q_{\text {Ltot }}(\ell+1)}{Q_{\text {Gtot }}(\ell+1)}, \quad 0 \leq \alpha_{Q} \leq 1 .
$$

Correspondingly, each active node generates a reactive power reference of:

$$
Q_{G n}^{*}(\ell+1)=\alpha_{Q} \cdot Q_{G n}^{\max }(\ell+1) .
$$

2) $Q_{\text {Ltot }}(\ell+1)>Q_{G t o t}^{\max }(\ell+1)$ : in this case, the desired reactive power can be generated through a controlled overload of the electronic power interfaces. In this case we set:

$$
\alpha_{Q}=1+\frac{Q_{\text {Ltot }}(\ell+1)-Q_{G t o t}^{\max }(\ell+1)}{Q_{\text {Gtot }}^{\text {over }}(\ell+1)-Q_{\text {Gtot }}^{\max }(\ell+1)}, \quad 1<\alpha_{Q} \leq 2 .
$$

Correspondingly, each active node generates a reactive power reference:

$$
\begin{aligned}
Q_{G n}^{*}(\ell+1) & =Q_{G n}^{\max }(\ell+1)+ \\
& +\left(\alpha_{Q}-1\right)\left(Q_{G n}^{\text {over }}(\ell+1)-Q_{G n}^{\max }(\ell+1)\right),
\end{aligned}
$$

The $\alpha_{P}$ and $\alpha_{Q}$ coefficients allows the calculation of the power reference at the EGs. In compact form, for all the cases above it holds:

$$
\begin{aligned}
P_{G n}^{*}(\ell+1) & =P_{G n}^{\min }+\left(P_{G n}-P_{G n}^{\min }\right) \cdot \min \left(\alpha_{P}, 1\right)+ \\
& +\left(P_{G n}^{\max }-P_{G n}\right) \cdot \max \left(\alpha_{P}-1,0\right), \\
Q_{G n}^{*}(\ell+1) & =Q_{G n}^{\max } \cdot \min \left(\alpha_{Q}, 1\right)+ \\
& +\left(Q_{G n}^{\text {over }}-Q_{G n}^{\max }\right) \cdot \max \left(\alpha_{Q}-1,0\right),
\end{aligned}
$$

\section{Grid connected operation}

In this case the UI is turned off, and the PCC delivers all the required power which is not provided by the local generators. In particular, as observed above, the control is non-critical since the mains ensure the power balance. The control master may ask the EGs to deliver any power level within their capacity (depending on the type of power source). For wind turbines or PV plants the best solution is to fully exploit their renewable energy, while for other types of sources (small hydro, fuel-cells, gas turbines) cost issues must be considered. In any event, the EGs can feed reactive power to demanding loads, thus reducing distribution losses, improving node voltage stability, and increasing the power factor at the PCC. Upon request from the UI, the EGs can also deliver or absorb more active power, at the expense of the energy reserve in their energy storage units. This can be done to meet internal needs such as node voltage stabilization, current limitation in the feeders or to respond to power demand from the utility.

The control strategy of Sections III-A and III-B can be directly adapted to the on-grid case. Indeed, setting $P_{\text {Ltot }}(\ell+$ $1)=P_{\text {Gtot }}(\ell+1)$, we get $\alpha_{P}=1$, forcing the distributed sources to inject all the power they generate into the grid. Note that, we may overestimate the amount of generated power, $P_{\text {Gtot }}(\ell+1)$, to accommodate several factors such as the amount of power needed to restore the state of charge of the local ESs. In any case, the UI provides the power required to maintain the balance between generated and absorbed powers. The UI may contribute to the reactive power compensation by computing $Q_{G 0}$ in (7), and injecting the corresponding reactive power $Q_{\text {Ltot }}(\ell+1)$ in the next cycle $\ell+1$.

\section{RESUlTS}

In this section, we discuss the performance of the proposed control strategy considering the power microgrid of Section II. The system, in terms of control, energy production and power demand, evolves in slotted time, where the slot duration is $T_{S}=1$ minute. Before delving into the analysis of the results, in the following paragraphs, we briefly discuss the considered statistical models for the renewable energy sources and the end-user demand.

Also, we provide insights for a proper sizing of the UI when operated in islanded mode. To this end, we assume a UI with infinite generation and storage capabilities, and operate the microgrid in islanded mode. By observing the requirements on the UI, we are then able to infer its sizing in a realistic deployment.

Renewable source model: energy traces for the photovoltaic sources have been obtained using the SolartStat tool [13]. In detail, energy generation statistics (cumulative distribution functions, cdf) have been generated for each month of the year and for each hour of the day for the city of Los Angeles. For the solar modules, we have considered the Panasonic N235B solar panel technology, accounting for a surface of about $10 \mathrm{~m}^{2}$ (delivering a nominal power of about $4 \mathrm{~kW}$ ). The solar modules have a tilt angle of $45^{\circ}$ and an azimuthal displacement, with respect to the real South, of $30^{\circ}$. Hence, 


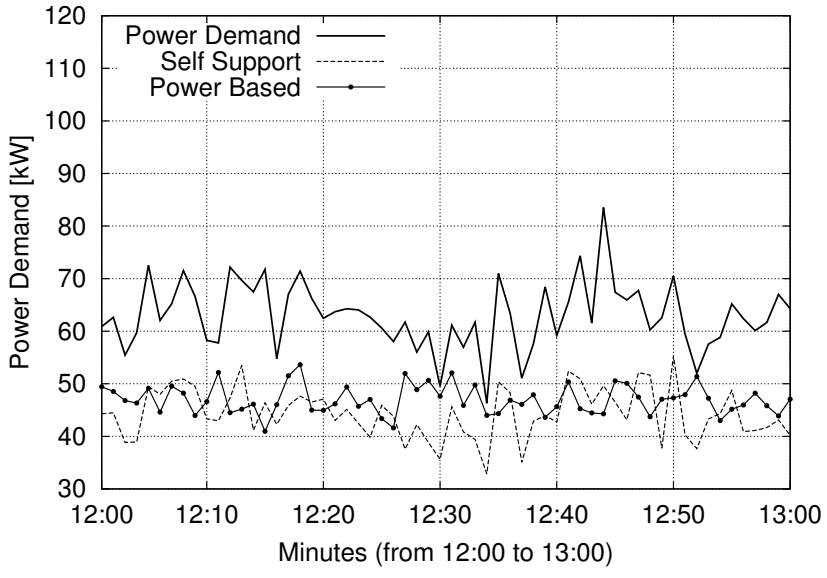

Fig. 2. Total power demand at the UI for a typical day of April.

these cdfs have been utilized to generate the current harvested from each solar module with a time granularity of $T_{S}$.

Modeling power demand: an accurate statistical model has been derived from the household electric power consumption data set, available at [14]. This database contains fine-grained (one per minute) measurements of active and reactive power demand from residential structures, collected between December 2006 and November 2010. Following the approach of [13], we have obtained power demand cdfs for each month of the year, day of the week and hour of the day. A power demand process is then updated for each end-user every $T_{S}$ seconds according to these statistics. Note that although the same cdf is considered for different end-users in the same time slot, their demands are independently drawn from this cdf.

Performance analysis: for comparison purposes, we introduce a simple algorithm, referred to as Self Support, where each EG makes local decisions without interacting with the UI. In detail, the highest priority of each EG corresponds to using the harvested energy to feed the local load. The excess energy, if any, is used to charge the local battery and the residual energy is injected into the grid.

Figs. 2 and 3 show the total power demand at the UI when the Self Support algorithm and the proposed control solution (referred to as Power Based) are used. In Fig. 4, we instead show the corresponding average state of charge of the local ESs. Moreover, we show the performance when no energy production / injection is accounted for (referred to in the plots as Power Demand). For these results, each active user has a battery with capacity of $28 \mathrm{Ah}$ operating at $240 \mathrm{~V}$. When the harvested energy is abundant (see Fig. 2), the proposed algorithm performs similarly to Self Support. As shown in Fig. 4, in April, Self Support and Power Based provide peak leveling while also charging the local batteries. From Fig. 3 we see that, as expected, the total demand is increased in December. In this case, the harvested energy is insufficient to fulfill the end-user demand and the distributed energy storage is utilized to support the loads and perform peak leveling. Power Demand accomplishes this task quite successfully, leveling the total demand at around $P_{G 0}(\ell)=40 \mathrm{~kW}$ (that is an

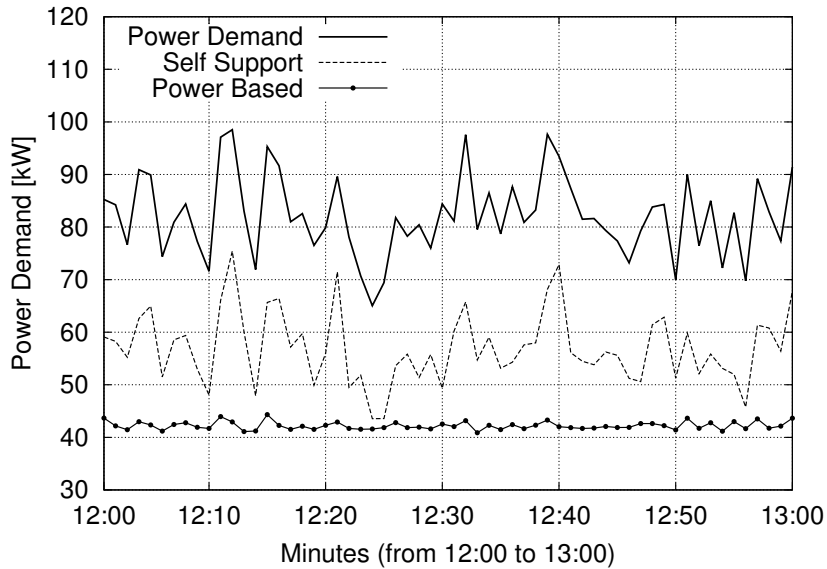

Fig. 3. Total power demand at the UI for a typical day of December.

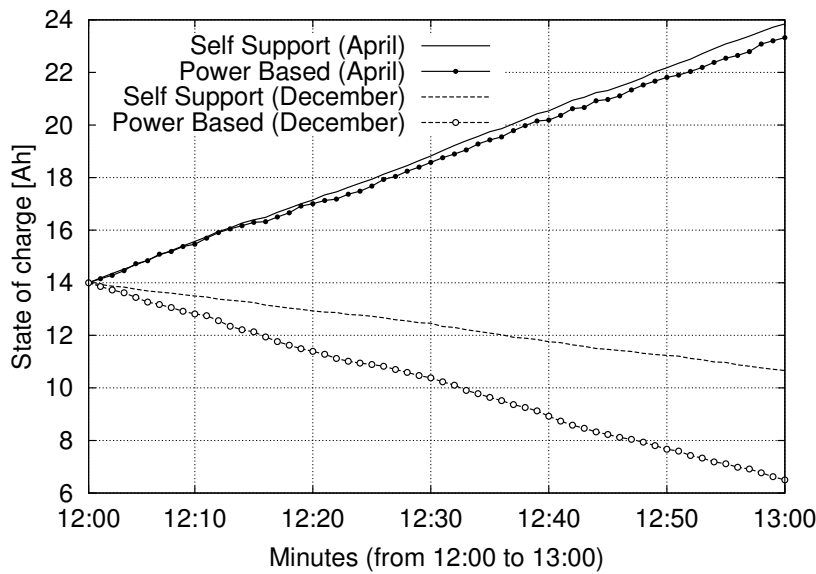

Fig. 4. Average state of charge for an end-user storage unit (battery).

input parameter for the algorithm). The total power demand is thus more than halved at the expense (see Fig. 4) of a reduced energy reserve at the end-users. Note that our algorithm can effectively level the required power as long as there is some residual charge in the distributed batteries. In the considered example, the state of charge decreases of about $8 \mathrm{Ah}$ in an hour. This means that, considering an ideal behavior for the discharge process, the adopted storage units can guarantee a full support for about 3.5 hours in the considered setup (i.e, number of active users and reference value $P_{G 0}(\ell)$ ).

The results of Figs. 5 and 6 are obtained as follows. We have considered a typical day of April and obtained the power demand and energy generation traces for all users (one sample per slot per trace). Thus, for each time slot, we have computed the difference between the total demand and the generated energy. The temporal average of this time series has been then used as the reference value $P_{G 0}(\ell) \approx 15 \mathrm{~kW}$ for the Power Based algorithm. Thus, we have run the Power Based algorithm for this same day, for these same traces and by assuming a sufficiently large battery capacity (e.g., 200Ah at $240 \mathrm{~V}$ ) at each active user, so that the performance of the 


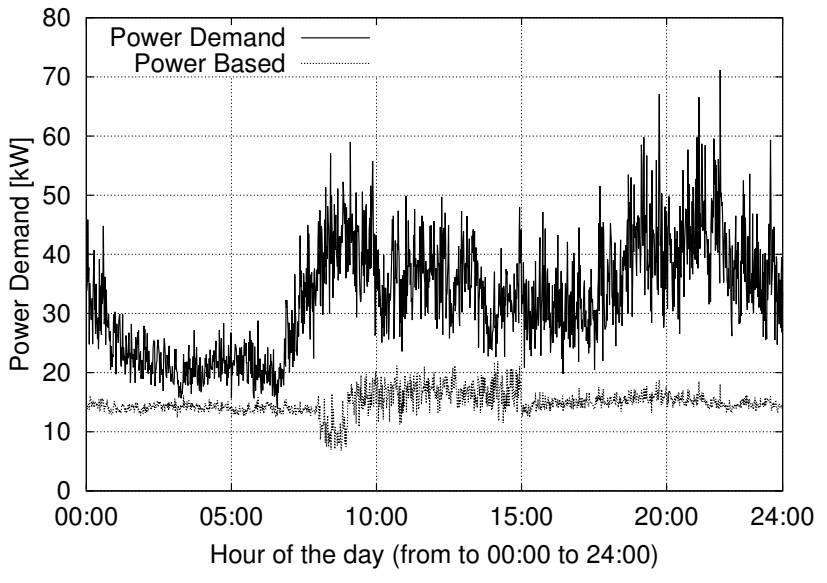

Fig. 5. Total power demand at the UI for a typical day of April.

algorithm will not be affected by it. The rationale behind this is that we are trying to operate the network so that the minimum possible amount of power is required from the UI, by exploiting as much as possible the distributed energy generation capability of the active users. Fig. 5 shows the results of this experiment for a typical April's day and we see that Power Based effectively accomplishes the task of leveling the power demand around $P_{G 0}(\ell) \approx 15 \mathrm{~kW}$. We also observe that Power Based has a somewhat bimodal behavior: i) for a small energy (before 8 a.m. and after 3 p.m.) a more conservative behaviour is observed, slightly relaxing the total demand with respect to the target $P_{G 0}(\ell)$; ii) when the energy income is abundant (between 8 a.m. and 3 p.m.) a better peak leveling is observed, taking advantage of (and lowering) the distributed energy reserve. Fig. 6 shows the state of charge of the battery of a typical end-user during the entire day. As seen from this plot, the required capacity is quite high and impractical due to economical and technological arguments. However, that capacity would assure the maximum exploitation of the energy production capabilities in the considered settings. As future avenues of research, note that increasing the number of active nodes would decrease the required capacity and we may also put a cap on it and check how that affects the performance as a function of the various system parameters.

\section{CONCLusions}

In this paper we have proposed a lightweight and effective algorithm for the energy management of prosumer communities. This algorithm provides satisfactory results, fulfilling its design objectives at the expense of a truly limited communication overhead (the transmission of a pair of reference values to each active user per time slot). We shed some light on battery sizing showing that, given a proper dimensioning of the storage capacity, self-sustainability is indeed possible and the proposed approach is able to reduce the total power required to the mains from a minimum of one half to a maximum of one sixth for the considered network setup. These figures, as well as the required storage capacity at the end-user side, highly depend on the number of users with energy harvesting and

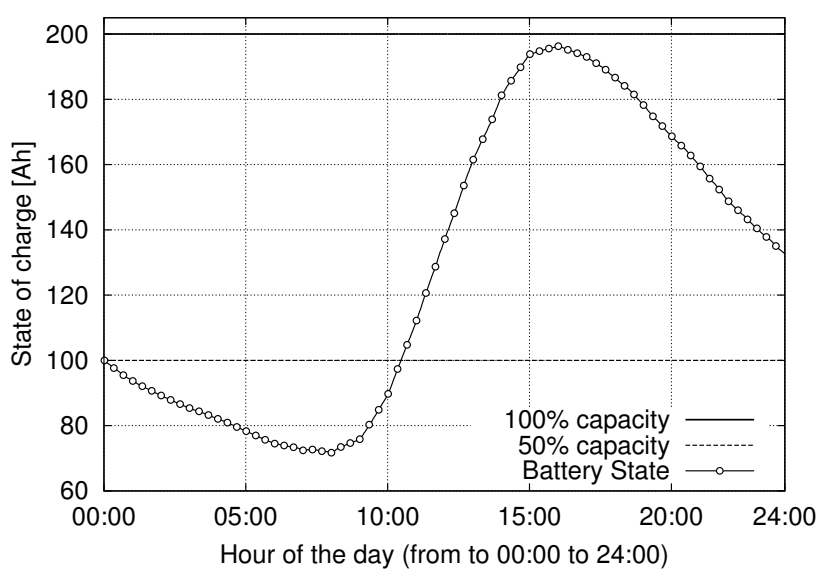

Fig. 6. State of charge for a typical end-user in the month of April.

storage capabilities. A more detailed study of these tradeoffs is left as a future work.

\section{REFERENCES}

[1] L. Tao, C. Schwaegerl, P. Mancarella, G. Strbac, N. Hatziargyriou, and B. Buchholz, "European Roadmap for Microgrids," in Proc. CIGRE, Paris, France, Aug. 2010.

[2] R. Ipakchi and F. Albuyeh, "Grid of the future," IEEE Power and Energy Magazine, vol. 7, no. 2, pp. 55-62, Feb. 2009.

[3] H. Farhangi, "The path of the smart grid," IEEE Power and Energy Magazine, vol. 8, no. 1, pp. 18-28, Jan.-Feb. 2010.

[4] J. Rocabert, G. Azevedo, A. Luna, J. Guerrero, J. Candela, and P. Rodriguez, "Intelligent connection agent for three-phase grid-connected microgrids," IEEE Trans. Power Electronics, vol. 26, no. 10, pp. 2993 3005, Oct. 2011.

[5] R. Anderson, A. Boulanger, W. Powell, and W. Scott, "Adaptive stochastic control for the smart grid," IEEE Proceedings, vol. 99, no. 6, pp. 1098-1115, May 2011.

[6] A. Dimeas and N. Hatziargyriou, "Operation of a multiagent system for microgrid control," IEEE Trans. Power Systems, vol. 20, no. 3, pp. 1447-1455, Aug. 2005.

[7] J.-Y. Kim, J.-H. Jeon, S.-K. Kim, C. Cho, J. H. Park, H.-M. Kim, and K.-Y. Nam, "Cooperative control strategy of energy storage system and microsources for stabilizing the microgrid during islanded operation," IEEE Trans. Power Electronics, vol. 25, no. 12, pp. 3037-3048, Dec. 2010.

[8] A. Mohsenian-Rad, V. Wong, J. Jatskevich, R. Schober, and A. LeonGarcia, "Autonomous demand-side management based on gametheoretic energy consumption scheduling for the future smart grid," IEEE Trans. Smart Grids, vol. 1, no. 3, pp. 320-331, Nov. 2010.

[9] D. Forner, T. Erseghe, S. Tomasin, and P. Tenti, "On efficient use of local sources in smart grids with power quality constraints," in Proc. First IEEE Int. Conf. on Smart Grid Commun. (SmartGridComm), 2010, pp. 555-560.

[10] T. Erseghe and S. Tomasin, "Power flow optimization for smart microgrids by sdp relaxation on linear networks," IEEE Trans. Smart Grid, vol. 4, no. 2, pp. 751-762, 2013.

[11] E. Serban and H. Serban, "A control strategy for a distributed power generation microgrid application with voltage- and current-controlled source converter," IEEE Trans. Power Electronics, vol. 25, no. 12, pp. 2981-2992, Dec. 2010.

[12] P. Tenti, H. K. Morales, and P. Mattavelli, "Conservative Power Theory, a Framework to Approach Control and Accountability Issues in Smart Microgrids," IEEE Trans. Power Electronics, vol. 26, no. 3, pp. 664-673, May 2011.

[13] M. Miozzo, D. Zordan, P. Dini, and M. Rossi, "SolarStat: Modeling photovoltaic sources through stochastic markov processes," in Proc. IEEE ENERGYCON, Dubrovnik, Croatia, May 2014.

[14] K. Bache and M. Lichman, "UCI Machine Learning Repository," 2013. [Online]. Available: http://archive.ics.uci.edu/ml 